\title{
LARGE SCREEN DISPLAY FOR THE MISSION CONTROL CENTER
}

\author{
Martin J. Skudlarek \\ Ford Aerospace Corporation, Space Information Systems Operation \\ MS B2E, P. O. Box 58487, Houston, Texas 77258-0487
}

\section{INTRODUCTION}

The Mission Control Center (MCC), located at the Johnson Space Center near Houston, Texas, is the primary point of control and monitoring for National Space Transportation System (NSTS) flight activities. NSTS flight managers monitor and command spacecraft from one of two Flight Control Rooms (FCR). Each FCR is equipped with five large screen displays for group dissemination of spacecraft system status and vehicle position relative to Earth geography. The primary or center screen display is ten feet in height and twenty feet in width. The secondary or side screens are seven and one-half feet high and ten feet wide. The center screen projection system is exhibiting high maintenance costs and is considered to be in wear-out phase.

The replacement of the large center screen displays at the MCC is complicated by the unique requirements of the Flight Controller user. These requirements demand a very high performance, multiple color projection system capable of the display of high resolution text, graphics and images produced in near real time. This paper describes the current system to be replaced, the replacement system requirements, the efforts necessary to procure the major element of this system (the projector) for the government, and how the new capabilities are to be integrated into the existing MCC operational configuration.

\section{OVERVIEW OF EXISTING SYSTEM}

The current center screen projection system represents the state-of-the-practice for electro-optical systems in the early half of the 1960 's. This rear screen based projection system is comprised of three major subsystems; the projector, mirror and screen, and the driving electronics.

\section{The projector}

Manufactured by LTV Corporation, the projector subsystem utilizes seven individual xenon lamp/slide assemblies, mounted on a common structure, to form composite images on the screen. (See figure 1.) Each assembly performs a specific function and by way of color filtering, each can provide a specific color. Basic slides are constructed of glass, with a metal film coating, a few microns thick, to provide opaqueness, and are mounted to a metal frame.

Five of the seven projectors are equipped with carousels to provide a supply and repository for new and used slides respectively. Four of those five projectors, referred to as plotters, are equipped with diamond tipped scribes driven from $X / Y$ servos, which scrape off the metal coating, allowing light to pass through the slide. The two projectors without carousels, spotters, utilize slides prepared with artwork, whose subject (i.e., shuttle outline) may be positioned within the frame of the image by $\mathrm{X} / \mathrm{Y}$ servos similar to the scribing mechanism. The remaining carousel projector, background, displays a static background from prepared artwork (i.e., map). To prevent damage to the slides from the heat produced by the close proximity of the xenon lamps to the slides, compressed air must be forced onto the slide surface. When simultaneously illuminated, color modified through filters, and modulated by art work or scribed slides, the light from these individual projector assemblies is integrated on the screen to develop the familiar "world map" image.

\section{The Mirror and Screen}

Due to a floor space constraint (see figure 2), and long throw distance requirement of the projector, the optical path to the center screen must be folded. This is accomplished through the utilization of a large front surface reflection system. This system consists of two five by six and one half foot pieces of front surface aluminum coating number 749 glass. Glass mounting 


\section{ORIGINAL PAGE \\ BLACK AND WHITE PHOTOGRAFH}

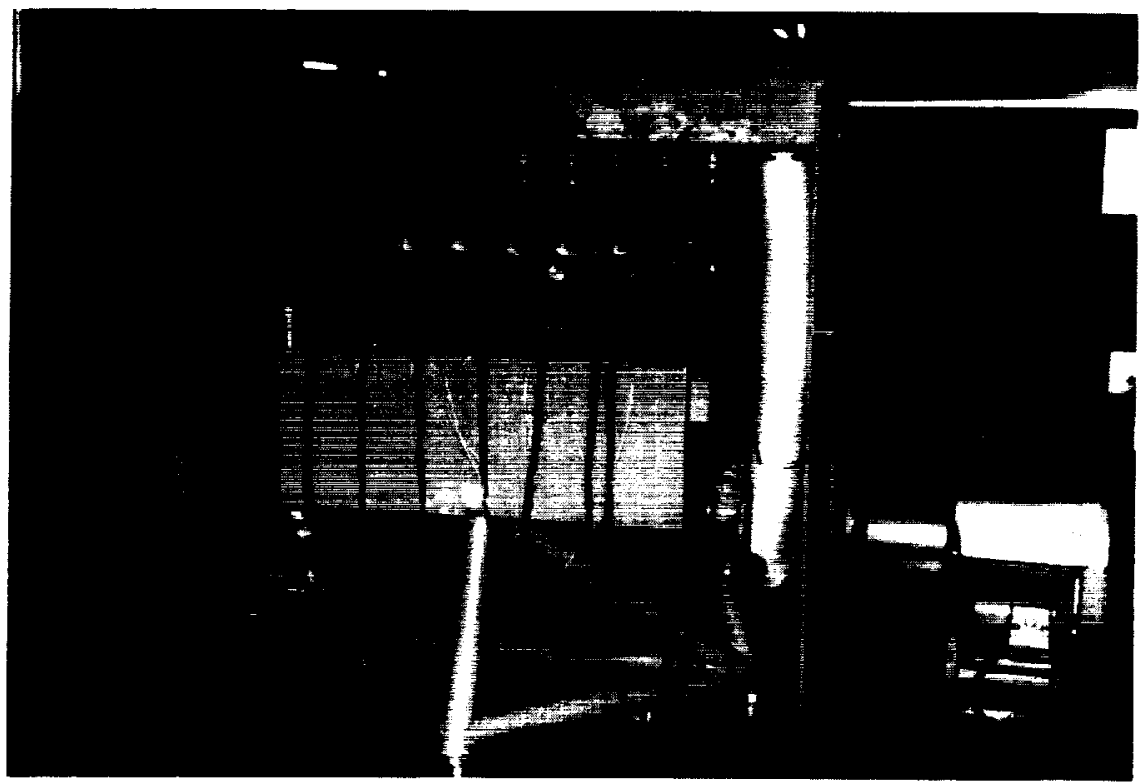

Figure 1. Existing LTV/Ford Aerospace center screen projector.

structures allow for tilt and azimuth adjustment. The support structure for the glass and glass mounts elevates the entire assembly to the required height of eleven feet, centerline.

The viewing screen is a single ten foot, one inch, by twenty foot, one inch, by 0.375 -inch sheet of coated glass. The rear-projection coating faces the viewers.

\section{Driving Electronics}

Ford Aerospace specially designed and built the electronics to command the projector because of the unique nature of the projector interfacing requirement, for example; when to change slides or color. Nine standard equipment racks, six feet in height, house the interface electronics and xenon lamp power supplies. Commands are received from the mission operations mainframe computers and translated into analog voltages for the scribing pen or slide positioning servos. The logic is at a five inch by five inch card level. Each card performs a logic function, i.e., NAND gate. Physical fatigue is reducing the reliability and availability of this interface. Due to a sagging card cage support structure, the logic cards become unseated from their edge connectors and even with redundant channels, the subsystem has a high failure rate.

\section{NEW SYSTEM REQUIREMENTS}

Despite the complexities and short comings discussed to this point, the current system produces extremely sharp, bright, functional displays to which the user has become accustomed. Defining realistic and achievable requirements for a replacement system to match the current system's capabilities has been a difficult task. As will be discussed in a subsequent section, several iterations to generate requirements, release Request for Proposals (RFP), and evaluation of submitted proposals were necessary to finally achieve a successful procurement. The requirements delineated below correspond to the projector to be manufactured under the current subcontract. In most major procurements for the government, a committee is usually assembled to define system requirements. This project was not unique in that regard.

\section{Display Characteristics}

The display shall fill the entire ten by twenty foot screen surface from a maximum throw distance of not more than 35 feet. Provide an average illumination of 2500 lumens, flat modulated white light, 30 to 1 contrast ratio, uniform across the entire screen within $\pm 25 \%$ as measured from the rear of the screen with a standard photometer. 


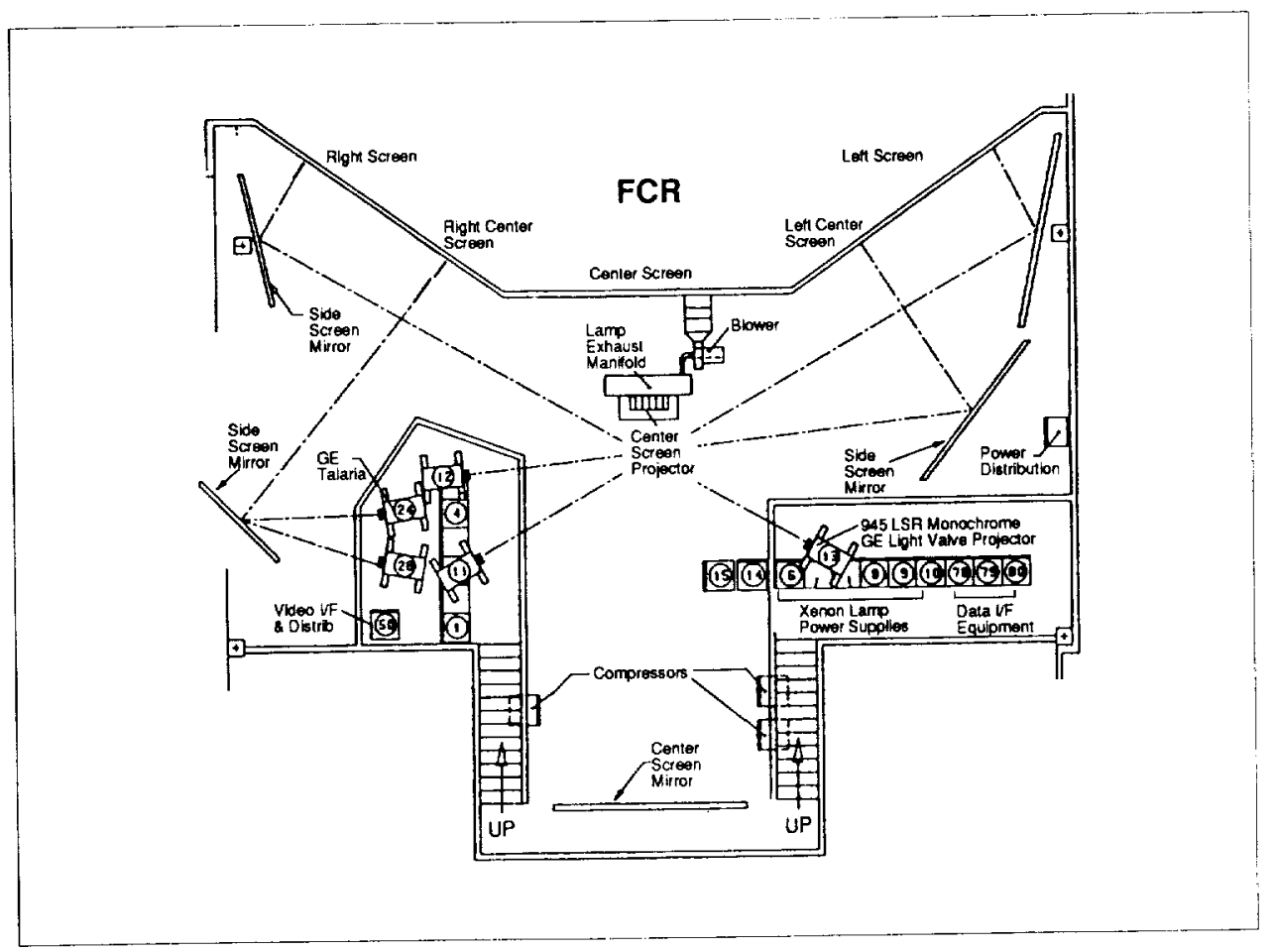

Figure 2. FCR projection room layout.

The projector shall be compatible with the analog RGB video output of several sources, such as; non-interlaced high resolution engineering workstations, the current 945 line scan rate repeat field monochrome video system and standard RS-170A color video. The projector shall have a "sense and select" automatic scan rate lock capability for a predetermined range of the possible video input scan rates designated for its use. The projector shall also be capable of accepting an extremely high resolution input $(1800 \times 900 \times 60)$ non-interlaced, wide aspect signal for use as a center screen replacement projector.

The projector shall exhibit geometric distortion less than or equal to 0.5 percent of the screen height in a circle with origin at the center of the screen, with a diameter equal to the screen height, and less than 1 percent outside of that circle. Primary color registration shall be within 0.1 percent of screen height.

\section{Commonality}

To provide for economies of scale in initial procurement and in logistical support considerations, commonality is an important requirement. It is planned that, not only the projector, but the workstation/graphics processor driving the projector shall be common elements in many other manned-mission support disciplines. In the FCR two additional projectors are planned to satisfy the secondary or side screen replacement requirements. There are several new manned-spaceflight control facilities in the initial stages of development. These centers are expected to require large screen displays:

- Space Station Freedom Control Center

- Orbital Maneuvering Vehicle Control

- Crew Emergency Return Vehicle Control.

Additionally, space mission simulators require high light output, extremely high resolution projectors for simulation of ascent, on-orbit, and entry activities. The Shuttle Mission Training Facility is currently undergoing an upgrade and plans are being drawn up for the Space Station Freedom Training Facility. In all, over twenty projection systems may be required in the next two to five year period.

\section{PROCUREMENT OVERVIEW}

The requirements committee initially defined requirements for a projector to satisfy a center screen replacement in 1984. It was learned, after an RFP cycle and some fact finding, that such a projector was not available commercially. Subsequently, the Government determined that procurement of side screen replacement projectors could be supported by commercially available products. The requirements were modified to reflect the needs of the side screen replacement systems. In essence, these requirements are to display information similar to that available on a FCR nineteen inch engineering workstation CRT. The RFP was released in late 1985 and fact finding commenced in early 1986. 
Fact finding consisted of visits to various supplier's manufacturing facilities, and demonstrations in the FCR itself. Six qualified bidders who met the specifications as written were selected. Ford Aerospace recommended, however, that none of the respondents offered a product satisfactory to the users requirements and that the procurement effort should be terminated.

Considerable experience had been amassed in the previous procurement attempts. An understanding of the large screen display state-of-the-practice had been acquired by the government and Ford Aerospace. Also, the MCC user community was educated to the fact that large screen display devices had significant display restrictions over CRTs and that the best quality display available would be expensive to acquire. Considering all lessons learned, a specification was developed that, if met, would satisfy a wide range of requirements (see above). Sufficient funding had been allocated to allow for the development of augmentations, modifications or upgrades to commercial products, if necessary, to satisfy the known requirements. In late 1986, an RFP was released defining such a projector. Six respondents submitted proposals of which two were determined to be in a competitive range. The six respondents were categorized as follows; two were direct laser projection products; two were Oil-film based projectors; and, two were based upon the polarizing light characteristics of crystals. The demonstration and fact finding procedures clearly identified the best proposal and candidate projector. The candidate selected was the Hughes Aircraft Company, Ground Systems Group/Fullerton, HDP-6000B projector, a liquid crystal based product.

Ford Aerospace is currently engaged with Hughes in negotiations to provide enhancements (see 6.0) to the original HDP-6000B projector. The two projectors currently subcontracted are scheduled to be delivered to Ford Aerospace in July of 1990. Production of the two HDP-6000B units will commence in February of 1989 at the Hughes Industrial Products Division in Carlsbad, CA.

\section{PROOF-OF-CONCEPT}

Given the level of funding required to purchase two large screen display projectors of this unique nature, NASA development managers desired a "check point" or Proof-of-Concept (POC) demonstration to ascertain if the projector could perform the assigned task adequately. Ford Aerospace included in the subcontract to Hughes the provisions for such a demonstration at the MCC FCR facility. Previous demonstrations, as part of procurement fact finding, yielded comments from the MCC user community expressing concern about insufficient illumination and display size. The subcontract included a clause that provides a POC demonstration of the specified brightness and display size prior to commencement of production.

Hughes modified a "brassboard" prototype projector to satisfy the POC requirements. The standard Arc lamp reflector was modified to produce twice the light output. The Xenon lamp size was increased to 2500 watts from 1000 watts. The scan circuitry was modified to accept video signals from the graphics processor selected for use by Ford Aerospace during the POC.

Ford Aerospace acquired a Sun $3 / 160$ workstation to serve as the workstation host for the graphics processor and application software necessary to provide and control video signals to the POC projector. The application software had been under development at Ford Aerospace for approximately a year and consisted of nearly twenty thousand lines of Unix based $C$ language code. The graphics processor, used for POC, was a Parallax 1280 series VME board set with RS$170 \mathrm{~A}$ video overlay capability. The Parallax was chosen for its RGB video output timing flexibility and TV overlay capabilities. By using the flexible video output timing characteristics of the Parallax the development team was able to match the maximum possible scan

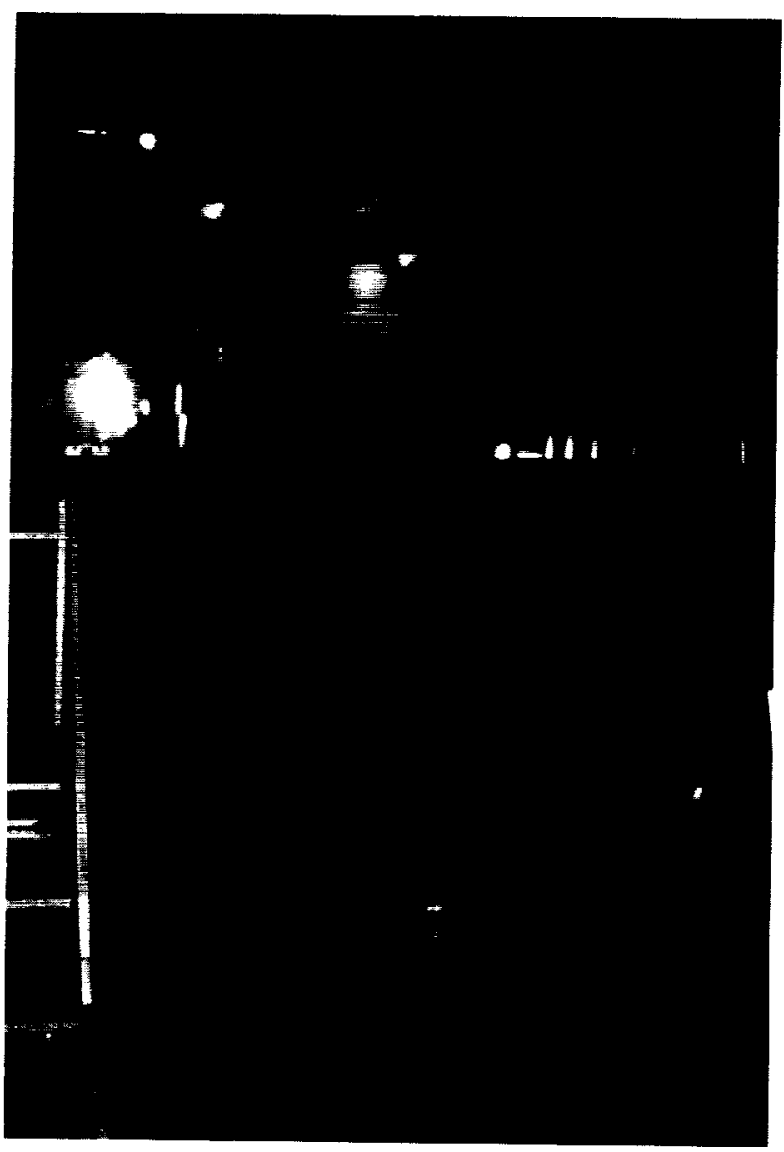

Figure 3. Proof-of-Concept Projector, lift table and Sun 3/160 workstation. 
rate available from the POC projector, thereby, providing the optimum quality obtainable from the POC projector's electronics package reducing the overall cost of the subcontract to the government. It would have been prohibitively expensive and self defeating to produce a full production projector for POC. A series of four integration tests was preformed over a six month period at Hughes to define the POC projector's operational scan limitations and to integrate, test and align the POC system.

Hughes personnel installed the POC projector in the FCR projection room on a hydraulic lift table (see figue 3) provided by Ford Aerospace specifically for the POC. The lift table allowed placement of the POC projector's output optics at the center line of the screen. The workstation was configured so that the user interface hardware was on the user side of the screen and the workstation CPU/Graphics Processor combination was located adjacent to the projector. After an extensive checkout, alignment and test cycle, a performance verification test was preformed to contractually verify POC performance. The modulated light output, measured from the rear of the screen by a photometer, at nine locations adjacent and perpendicular to the screen surface, was over 2800 lumens. It was also determined that the projector, when located an additional two feet further from the screen, would provide a full ten by twenty screen display. (POC size was set at ten by eighteen feet due to the existing system's mirror support structure placement.)
The POC was considered to be successful by a majority of the MCC user community, NASA development managers, and the Ford Aerospace development team. (See figure 4.) A questionnaire was distributed during the user demonstration segment of the POC which asked for the user's opinion of the suitability of the demonstrated system as a replacement for the existing system. Over eighty percent of respondents to the questionnaire responded favorably. Consequently, NASA has directed Ford Aerospace to continue in our efforts to replace the center screen systems and investigate the commonality candidates.

\section{ENHANCEMENTS}

From the user comments during POC and NASA development manager's inputs, it was determined that further enhancements should be incorporated into the HDP-6000B production projectors. A brief description of the proposed enhancements follows.

\section{Scan Circuits to $68 \mathrm{KHz}$}

This enhancement provides for a higher level of commonality between various projector requirements, ease of manufacture, and logistical sparing. With the enhanced version of scan electronics, virtually any engineering class workstation, or Advanced Television source may be connected to the HDP-6000B inputs. This electronics package shall also be used in another Hughes program.

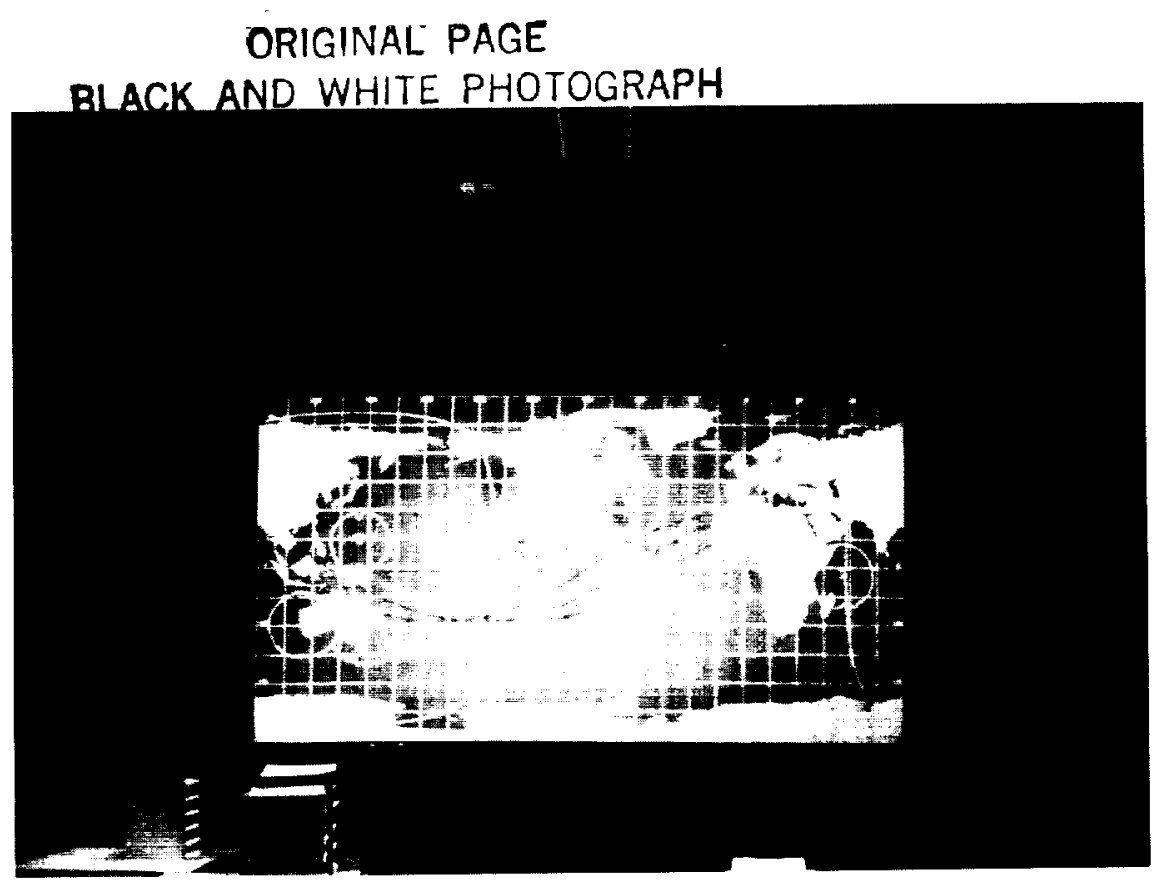

Figure 4. POC demonstration display of the "World Map."

ORIGINAL PAEE IS OF POOR QUALE: 


\section{Video Bandwidth to $110 \mathrm{MHz}$}

Given a resolution requirement for the center screen of $1800 \times 900 \times 60$ non-interlaced, it was necessary to increase the video bandwidth (sharpen pixel rise/fall times) in the production version of the HDP-6000B.

\section{FUTURE PLANS}

The existing subcontract shall be modified to include the enhancements discussed in the previous paragraphs. Hughes shall initiate production of the two HDP-6000B projectors shortly thereafter. As the actual fabrication of the two production HDP-6000B projectors is in progress, Ford Aerospace shall be monitoring that effort, in addition to developing the telemetry/trajectory data to video signal processing and generation software and equipment.

\section{Workstation}

The final host platform for the world map generation application has yet to be identified. Once the projector input requirements are set, a procurement cycle will be initiated for the graphics processor to satisfy the high resolution display requirements and match the video signal timing requirements of the projector. As the candidates for the graphics processor are identified, analysis to identify the workstation to serve as host to the graphics processor and application software will commence. The processing speed of the individual workstation's flavor of Unix, native software development environment, internal bus structure and local area network commonality are prime considerations in the analysis process.

\section{Software}

As was stated previously, the application software development task had been proceeding in parallel to the hardware identification, procurement and development activities. In addition to generating and updating a world tracking map from data delivered to the application from spacecraft telemetry, the application must generate near real time graphical displays that convey spacecraft parameters during ascent and entry phases of the flight. At POC, a stable prototype of the application was available for use in the demonstration of the POC projector. The application was initially developed on a Masscomp 5600 class workstation. The Parallax, which is a VME based product, (Masscomp 5600s are Multibus based) required a port of the application software to the VME based Sun 3/160. Approximately $95 \%$ of the code transported transparently, with only the graphics specific calls in need of a rewrite. The original application uses the native graphics calls specific to the Masscomp workstation graphics processor and, in the case of the
Sun/Parallax, uses the Parallax native graphics instruction set.

POC provided a useful degree and quantity of feedback from the user community that simply could not be obtained from demonstrations of the application on a 19 inch CRT. Comments concerning character size were the most prevalent. Ford Aerospace intends to provide to NASA a human factors analysis on color and character usage for the large screen displays prior to the official final release of the application software.

\section{Installation}

Upon completion of fabrication, checkout and alignment of the projectors will be accomplished at the Hughes facilities prior to shipment. Upon receipt and receiving inspection by Ford Aerospace, the projectors will then be shipped to the Johnson Space Center, Building 30 , where they will be installed in the two FCRs. Each installation will consist of the projector, new screen, projector lift table, workstation/graphics processor, application software, maintenance monitors, maintenance documentation and the removal and subsequent surplus of the existing system. When the installation is complete, a final Acceptance Test will be preformed verifying the performance of each projector to specifications.

A trial period of non critical support may be required to acquaint the flight control personnel with the new system's capabilities and idiosyncrasies prior to the removal of the current system. This concept is facilitated by the projector/lift table configuration which allows the HDP-6000B to be raised/lowered, in/out of the optical path of the existing system for use of either system as the situation would warrant.

Ford Aerospace has included training, for the maintenance and operations contract personnel who operate and maintain all the flight support equipment for the $\mathrm{MCC}$, in the subcontract.

\section{CONCLUSIONS}

NASA and Ford Aerospace have selected what we believe is the optimum large screen display projector for our requirements. A methodology for the continuation of the system development and integration is in place and shall deliver the superior product NASA/ JSC is accustomed to.

\section{ACKNOWLEDGEMENTS}

The author wishes to thank NASA, Ford Aerospace, Hughes Aircraft Company, and Lamar Flanagan of NASA/JSC/FS73, for their support of this effort. This project is funded through NASA contract NAS9-15014. 